\title{
GENERALIZED KOLMOGOROV INEQUALITIES FOR MARTINGALES
}

\author{
by \\ David Gilat ${ }^{*}$ and William D. Sudderth ${ }^{* *}$ \\ Technical Report No. 257
}

\begin{abstract}
December, 1975
University of Minnesota

Minneapolis, Minnesota
\end{abstract}

\footnotetext{
* On leave from Te1-Aviv University.

${ }^{* *}$ Research supported by National Science Foundation Grant MPS75-06173.
} 


\section{ABSTRACT}

The classical Chebyshev inequality leads to an inequality for martingales which is often called the Kolmogorov inequality. It is shown here that many generalized Chebyshev inequalities for random variables lead in a similar way to martingale inequalities, and that the corresponding martingale inequality is sharp when the Chebyshev inequality is. 


\section{The main result.}

Let $R$ be the set of real numbers and let $B$ be the collection of Borel subsets of $R$. As is customary, set $R^{\infty}=R \times R \times \cdots$ and let $\beta^{\infty}$ be the product $\sigma$-field $\beta \times \beta_{3} \ldots$. Let $x_{1}, x_{2}, \ldots$ be the coordinate process on $R^{\infty}$. It is convenient here to regard a martingale as being a probability measure $P$ on $B^{\infty}$ under which the process $\left\{X_{n}\right\}$ is a martingale in the usual sense. There is no loss of generality since every martingale on an abstract probability space has a distribution for which $\left\{x_{n}\right\}$ is a martingale.

Next let $\Phi$ be a set of Borel functions from $R$ to $R$ and suppose every member of $\Phi$ is either convex or concave. Let $r$ be a mapping from $\Phi$ to $R$. Associate with $\Phi$ and $r$ the class $C=C(\Phi, r)$ of all probability measures $p$ on $\theta$ such that, for every $\varphi \in \Phi, \varphi$ is integrable with respect to $p$ and $\int_{\varphi p d p} \leq r(\varphi)$. Finally, let $M=M(C)$ be the collection of all probability measures $P$ on $\mathbb{R}^{\infty}$ such that $\left\{X_{n}\right\}$ is a martingale under $P$ and, such that, for every $n \geq 1$, the distribution of $X_{n}$ under $P$ is in $C$.

Theorem 1. If $B \in B$, then

$$
\begin{aligned}
\sup _{P \in M} P\left\{X_{n} \in B \text { for some } n\right\} & =\sup _{P \in M} P\left\{X_{1} \in B\right\} \\
& =\sup _{P \in C} P(B) .
\end{aligned}
$$

Proof: The second equality in ( 1 ) is trivial and it is obvious that the right-hand-side of the first equality is no larger than the left. It remains to prove the reverse inequality.

$$
\text { For } \begin{aligned}
x= & \left(x_{1}, x_{2}, \ldots\right) \in R^{\infty} \text {, let } \\
t(x) & =\text { least } n \text { (if any) such that } x_{n} \in B, \\
& =\infty \text { if there is no such } n .
\end{aligned}
$$


Then, for every $\mathbf{P}$,

$$
\begin{aligned}
P\left\{X_{n} \in B \text { for some } n\right\} & =P\{t<\infty\} \\
& =\lim _{\mathfrak{n} \rightarrow \infty} P\{t \leq n\} \\
& =\lim _{n \rightarrow \infty} P\left\{X_{t \wedge n} \in B\right\} .
\end{aligned}
$$

The proof will be finished once it is shown that, for $P \in M$, the distribution of $X_{\text {tan }}$ under $P$ is in $C$ and this latter fact is a consequence of the lemna below.

A stopping variable is a mapping $s$ from $R^{\infty}$ to the positive integers such that, for every $n$, the event $\{s \leq n\}$ is measurable with respect to $\left\{x_{1}, \ldots, x_{n}\right\}$

Lemma 1. If $P \in M$. and $s$ is a bounded stopping variable, then the distribution of $X_{s}$ under $P$ is in $C$.

Proof of Lemma 1: Let $\varphi \in \Phi$. Suppose first that $\varphi$ is concave. Then $\left\{\varphi\left(X_{n}\right)\right\}$ is a supermartingale under $P\left[3, V . T\right.$ 6,p.79] and $\int_{\varphi}\left(X_{s}\right) d P \leq$ $\int_{\varphi}\left(X_{1}\right) d P \leq r(\varphi)$, where the first inequality is by the optional sampling theorem [3,V.T 9,p.80] and the second is by definition of $M$. Suppose next that $\varphi$ is convex. Then $\left\{\varphi\left(x_{n}\right)\right\}$ is a submartingale under $P$ [3,V.T 6,P.79] and, if $m$ is a positive integer such that ssm, then again by the optional sampling theorem and the definition of $M$, $\int_{\varphi}\left(X_{s}\right) d P \leq \int_{\varphi}\left(X_{m}\right) d P \leq r(\varphi)$. This completes the proof of the lemma and of Theorem 1 .

Theorem 1 can be viewed as another reflection of the idea of bold play for gambling problems used in [1]. If a gambler, who seeks to attain a fortune in $B$, is allowed to select a martingale in the class $M$, 
then, according to Theorem 1 , he can come as near as is possible to reaching his goal at the first stage of play.

It is easy to check that the supremum in ( 1 ) is equal to $\sup _{P \in M} P\left\{\right.$ for some $n$ and all $\left.k \geq n, x_{k} \in B\right\}$

and also equal to

$$
\sup _{P \in M} P\left\{X_{n} \in B \text { for infinitely many } n\right\}
$$

Roughly, the reason is that, after reaching $B$, the process can be stopped and thus remain in $B$ from then on. This suggests the following generalisation of Theorem 1 .

Theorem 2. If $\forall$ is a nonnegative, Borel function on $R$, then

$$
\begin{aligned}
\sup _{P \in M^{l}}\left\{\left\{1 m_{n} \sup \psi\left(x_{n}\right)\right\} d P\right. & =\sup _{P \in M}\left\{\left\{1 i m_{n} i n f\left(x_{n}\right)\right\} d P\right. \\
& =\sup _{P \in M} \int \psi\left(x_{1}\right) d P \\
& =\sup _{p \in C} \int \psi d p .
\end{aligned} .
$$

Since Theorem 2 is not used in the examples of the next section, its proof is omitted. A proof can be based on the preceeding ideas together with Fatou's lemma and [5, Theorem 1]. Like Theorem 1, Theorem 2 has an interpretation for gambling problems. In fact, if $\phi$ is bounded and is regarded as being a utility function and if $\left\{x_{n}\right\}$ is viewed as the sequence of successive fortunes of a gambler, then $\int\left\{\lim _{n}\right.$ sup $\left.\psi\left(x_{n}\right)\right\} d P$ is the utility associated with the strategy $P$ by Dubins and Savage [6, Theorem 3.2]. 
Remark on semimartingales. If every function in $\Phi$ is either convex increasing or concave decreasing, then Theorems 1 and 2 remain true if, in the definition of $M$, "martingale" is replaced by "supermartingale or submartingale." The proofs are easily modified.

\section{Some applications.}

In this section Theorem 1 is applied to some known Chebyshev-type inequalities for real-valued random variables and the corresponding inequalities for martingales are obtained.

Examples 1 and $1^{\prime}$ present sharp upper bounds on the probability that an $\mathrm{L}_{1}$-bounded martingale ever leaves an open interval. Examples 2 and $2^{\prime}$ provide analogous results for $\mathrm{L}_{2}$-bounded martingales. The classical $\mathrm{L}_{1}$ and $L_{2}$ forms of Kolmogorov's inequality are special cases of Examples $1^{\prime}$ and $2^{\prime}$ respectively. In addition, these Examples also lead to onesided versions of the Kolmogorov inequality, some of which seem to be new. Finally, Example 3 considers the class of all martingales $\left\{x_{n}\right\}$ such that for each $n$, the Laplace transform of $x_{n}$ is majorized by the Laplace transform of the standard normal distribution. Such martingales may conveniently be called subnormal. Example 3 provides an upper bound on the probability that a subnormal martingale will ever exit from an open inter: val. A symmetric as well as a one-sided version of the inequality is also derived for this case.

Example 1. Fix a positive number $c$ and consider the class $c$ of all probability measures $p$ on $(R, \mathbb{B})$ with mean 0 and absolute first moment not exceeding $c$. Let $b$ and $a$ be positive numbers. Then according 
to an Inequality due to Glasser $[2, \mathrm{p} .481]$,

$$
\sup _{\mathrm{P} \in \mathrm{C}} \mathrm{P}\{(-\infty,-\mathrm{b}] \cup[a,+\infty)\}=\min \left\{1, \frac{\mathrm{c}}{2}\left(\frac{1}{\mathrm{~b}}+\frac{1}{\mathrm{a}}\right)\right\} .
$$

To adapt (3) to an application of Theorem 1 , take $\Phi=\left\{\varphi_{-}, \varphi_{+}, \varphi_{1}\right\}$. where $\varphi_{+}(x)=x=-\varphi_{-}(x)$ and $\varphi_{1}(x)=|x| ;$ set $r\left(\varphi_{-}\right)=0=r\left(\varphi_{+}\right)$and $r\left(\varphi_{1}\right)=c$, and let $B=(-\infty,-b] \cup[a,+\infty)$. Then apply Theorem 1 to (3) to obtain

$$
\sup _{P \in M} P\left\{X_{n} \leq-b \text { or } X_{n} \geq a \text { for some } n\right\}=\min \left\{1, \frac{c}{2}\left(\frac{1}{b}+\frac{1}{a}\right)\right\} \text {, }
$$

where $M=M(C)$ is the collection of all probability measures on $\left(R^{\infty}, \mathbb{R}^{\infty}\right)$ under which the coordinate process $\left\{x_{n}\right\}$ is a martingale with mean 0 and $\mathrm{L}_{1}$-norm bounded by $c$.

Thus if $\left\{X_{n}, n \geq 1\right\}$ is any $L_{1}$-bounded martingale with mean zero, then

$$
\text { Prob. }\left\{X_{n} \leq-b \text { or } X_{n} \geq a \text { for some } n\right\} \leq \frac{1}{2}\left(\frac{1}{b}+\frac{1}{a}\right) \text { sup } E\left|X_{n}\right| \text {. }
$$

Letting $b$ tend to to in (5) yields a one-sided version

$$
\text { Prob. }\left\{\sup X_{n} \geq a\right\} \leq \frac{1}{2 a} \sup E\left|x_{n}\right|
$$

which holds for martingales with mean zero.

Example $1^{\prime}$. This example differs from the preceeding one only in that the assumption of mean zero made there is omitted. Formally, let c>0 and take $C$ to be the class of all $p$ with absolute first moment no larger than $c$. Let $b$ and $a$ be positive numbers. The role played by (3) in Example 1 is here played by

$$
\sup _{p \in C} p\{(-\infty,-b] \cup[a,+\infty)\}=\min \left\{1, \frac{c}{m}\right\}
$$


where $m=\min \{a, b\}$. Since the indicator function of $\{x: x \leq-b$ or $x \geq a\}$ is dominated by $\mathrm{m}^{-1}|\mathrm{x}|$, it follows that the right-hand-side of (7) majorizes the left. In fact, the supremum in (7) is attained by a $p$ with support $\{-b, 0\}$ if $b=m$ and $\{0, a\}$ if $a=m$. (We were led to (7) by the general result of Karlin and Studden [2, Theorem 2.1,p.472].) Apply Theorem 1 with $\Phi=\{\varphi\}$ where $\varphi(x)=|x|$ and with $r(\varphi)=c$ to get Prob. $\left\{X_{n} \leq-b\right.$ or $X_{n} \geq a$ for some $\left.n\right\} \leq \frac{1}{m} \sup E\left|X_{n}\right|$. Set $a=b$ in (8) to obtain the classical Kolmogorov inequality. Let $b$ approach to in (8) to obtain the well-known inequality

$$
\text { Prob. }\left\{\sup x_{n} \geq a\right\} \leq \frac{1}{a} \sup E\left|x_{n}\right|
$$

In contrast to (5) and (6), which require the martingale $\left\{x_{n}\right\}$ to have mean zero, (8) and (9) hold for all $\mathrm{L}_{1}$-bounded martingales.

Example 2. Replace the condition on the first absolute moment of $p$ in Example 1 by a similar condition on the second moment. That is, consider the class $\mathrm{C}$ of $\mathrm{all} \mathrm{p}$ with mean 0 and variance at most $c$. Again, let $b$ and $a$ be fixed positive numbers and set $m=\min \{b, a\} . \quad B y$ Selberg's inequality $[2$, p. 475$]$,

$$
\begin{aligned}
& \sup _{\mathrm{P} F \mathrm{C}} \mathrm{P}\{(-\infty,-b] \cup[a,+\infty\}=1 \quad \text { if } a b \leq c \\
& =\frac{(b-a)^{2}+4 c}{(b+a)^{2}} \quad a b-m^{2} \leq 2 c<2 a b \\
& =\frac{c}{m^{2}+c} \quad 2 c<a b-m^{2} \text {. }
\end{aligned}
$$

Abbreviate the right-hand-side of $(10)$ by $U(a, b, c)$. Then Theorem 1 with 
the obvious $\Phi, r$ and $B$, yields

$$
\sup _{P \in M} P\left\{X_{n} \leq-b \text { or } X_{n} \geq a \text { for some } n\right\}=U(a, b, c) \text {, }
$$

where $M=M(C)$. Thus, for any $L_{2}$-bounded mean zero martingale,

$$
\text { Prob. }\left\{\mathrm{X}_{n} \leq-\mathrm{b} \text { or } \mathrm{X}_{n} \geq \text { a for some } n\right\} \leq U\left(a, b, \text { sup } \mathrm{EX}_{n}^{2}\right) \text {. }
$$

As $\mathrm{b} \rightarrow+\infty$, U reduces to $\mathrm{U}(\mathrm{a},+\infty, \mathrm{c})=\mathrm{U}(\mathrm{a}, \mathrm{c})=\frac{c}{\mathrm{a}^{2}+c}$. Hence, for martingales $\left\{x_{n}\right\}$ with mean zero,

$$
\text { Prob. }\left\{\text { sup } X_{n} \geq a\right\} \leq \frac{\sup E X_{n}^{2}}{a^{2}+\sup E X_{n}^{2}}
$$

Example 2*. Here, as in Example $1^{\prime}$, the mean zero assumption is dropped to obtain sharp bounds for all $\mathrm{L}_{2}$-bounded martingales. The notation here is the same as in Example $I^{\prime}$ except that $C$ is the set of $p$ with second moment no larger than $c$ and $\Phi=\{\varphi\}$ where $\varphi(x)=x^{2}$. The Chebyshev result corresponding to (7) is

$$
\sup _{p \in C} p\{(-\infty,-b] U[a,+\infty)\}=\min \left\{1, \frac{c}{m^{2}}\right\}
$$

The proof of (14) is similar to that of (7). By Theorem 1,

$$
\text { Prob. }\left\{X_{n} \leq-b \text { or } X_{n} \geq \text { a for some } n\right\} \leq \frac{1}{m^{2}} \text { sup } \operatorname{EX}_{n}^{2}
$$

for every $L_{2}$-bounded martingale $\left\{X_{n}\right\}$. Two well-known results can be obtained from (15) by taking $a=b$ for one and letting $b$ tend to $+\infty$ for the other.

Example 3. Suppose $p$ is a probabilicy measure on $(R, \beta)$ whose Laplace transform $\lambda_{p}(c)=\int^{c x} \mathrm{dp}(\mathrm{x})$ is finite for all real c. Bernstein's 
inequality

$$
p\{(-\infty,-b] U[a,+\infty)\} \leq \inf _{c>0} e^{-c b} \lambda_{p}(-c)+\inf _{c>0} e^{-c a} \lambda_{p}(c)
$$

is not hard to verify, or else its proof can be found, for example in $[4, p .86]$.

Consider now the class $\mathrm{C}=\mathrm{c}_{\lambda}$ of all $\mathrm{p}$ for which $\lambda_{\mathrm{p}}$ is majorized by some fixed function $\lambda$. Then when $\lambda_{p}$ is replaced by $\lambda$ on the right-hand-side of (16), an upper bound for the entire class $c_{\lambda}$ is obtained. Since for each $c$, the function $\varphi_{c}: x \rightarrow e^{c x}$ is convex, Theorem 1 , with $\Phi=\left\{\varphi_{c}: c \neq 0\right\}$ and $r: \varphi_{c} \rightarrow \lambda(c)$, applies to obtain the corresponding martingale inequality for the class $M_{\lambda}=M\left(C_{\lambda}\right)$. An interesting upper bound $(17)$ is thus obtained from (16) by taking $\lambda(c)=: e^{c^{2} / 2}=$ the Laplace transform of the standard normal distribution.

$$
\sup _{P \in M} P\left\{X_{n} \leq-b \text { or } X_{n} \geq a \text { for some } n\right\} \leq e^{-\frac{b^{2}}{2}}+e^{-\frac{a^{2}}{2}} \text {. }
$$

The symetric form of (17) as well as its one-sided version can be obtained as in the previous examples. We do not know whether the bound in (17) is the best possible.

Aknowledgment. Bert Fristedt helped us to discover the inequality (12) which was conjectured on the basis of the idea of bold play. 
References

1. Bubins, Lester E. and Savage, Leonard J. (1965). How to Gamble If You Must. Dover, New York.

2. Karlin, Samuel J. and Studden, William J. (1966). Tchebycheff systems: with applications in analysis and statistics. Interscience, New York.

3. Meyer, Paul A. (1966). Probability and Potentials. Blaisdell Publishing, Waltham, Mass.

4. Renyi, Alfred. (1970). Foundations of Probability. Holden-Day, San Francisco.

5. Sudderth, William D. (1971). A "Fatou equation" for randomly stopped variables. Ann. Math. Statist. 42 2143-2146.

6. (1971). On measurable gambling problems. Ann. Math. Statist. 42 260-269. 